\title{
Les femmes et l'Artisanat: Mythe et réalité
}

Jasleen Dhamija

Follow this and additional works at: https://knowledgecommons.popcouncil.org/departments_sbsr-pgy

Part of the Entrepreneurial and Small Business Operations Commons, Family, Life Course, and Society Commons, Gender and Sexuality Commons, International Public Health Commons, and the Regional Economics Commons How does access to this work benefit you? Let us know!

\section{Recommended Citation}

Dhamija, Jasleen. 1983. "Les femmes et l'Artisanat: Mythe et réalité," SEEDS no. 4. New York: Population Council. 


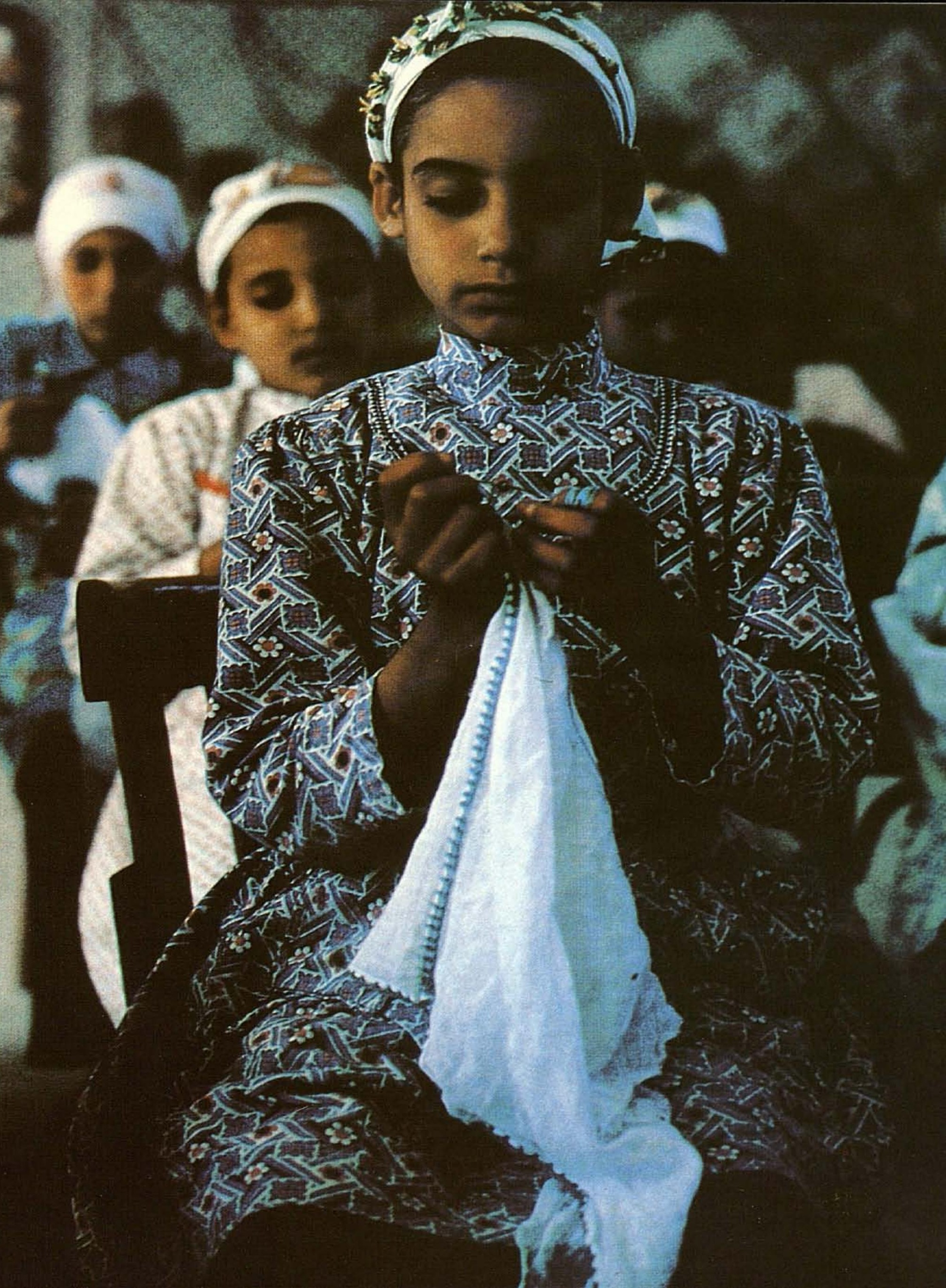


SEEDS est une série de brochures publiée en réponse aux demandes d'information venant du monde entier sur des idées de programmes innovatrices et pratiques, réalisées par et pour des femmes aux revenus faibles. Le but de ces brochures est de diffuser l'information et de stimuler la création de nouveaux projets, fondés sur les expériences positives de femmes qui travaillent pour améliorer leur propre statut économique et celui des autres. Les projets décrits dans ce numéro comme dans le reste de la série ont été choisis du fait qu'ils procurent aux femmes un revenu en espèce et les font participer activement aux prises de décisions comme aux profits. Ces projets sont bâtis suivant des critères économiques solides et permettent de surmonter avec succès des obstacles courants. Ces rapports n'ont cependant pas été conçus pour être suivis à la lettre car chaque effort de développement doit faire face à des ressources et des problèmes quelque peu différents. En revanche, ils relatent l'histoire d'une idée et sa mise en application avec l'espoir que les leçons apprises se révèleront utiles dans des environnements variés. C'est également pour être portés à l'attention de ceux qui détiennent les pouvoirs de décision qu'ils ont été rédigés et leur montrer que des projets générateurs de revenus, pour et par les femmes, sont viables et jouent un rôle important dans le développement.

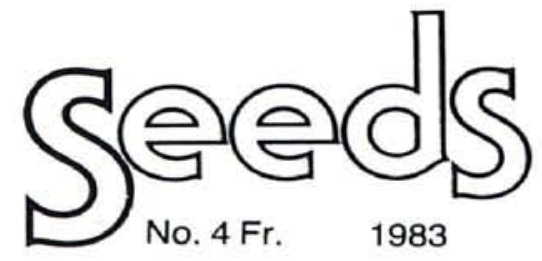

La traduction française de ce numero de SEEDS a été subventionnée par: Women in Development Office

Agency for International Development Washington, D.C. 20523 U.S.A. 


\title{
Les Femmes et L'Artisanat: Mythe et Réalité
}

\author{
par Jasleen Dhamija
}

\section{Introduction}

Chaque fois que des organisateurs, des responsables de programmes ou des directeurs de projets se posent la question: "Comment mettre en place et déverupper des activités pour les femmes qui soient viables et génératrices de revenus?" , la première chose qui leur vient à l'esprit est le travail manuel et l'artisanat. Le mythe est que ces derniers soient une occupation féminine, quelque chose que les femmes font bien, une activité qui, à priori, ne s'ingère pas dans leurs responsabilités ménagères, ne demande que peu d'investissement et une courte période de gestation. Rarement prend-t-on le temps d'examiner la réalité de la situation, aussi bien sous l'angle de l'industrie des travaux manuels que du point de vue de la vie des femmes que le projet se propose d'aider. Peu d'organisateurs ou d'équipes de programme se rendent compte que l'artisanat demande souvent une technique de spécialiste et des années d'apprentissage ou que les métiers aux revenus équitables sont déjà la propriété exclusive des hommes!

Le but de ce rapport est d'examiner les travaux manuels et l'artisanat comme un moyen d'assurer un revenu aux femmes. Parfois ces métiers représentent pour elles une importante source d'argent et leur permettent également de rester en lien avec leur héritage culturel propre. Dans la plupart des cas cependant, la production artisanale relègue les femmes dans un domaine où le travail est intense et exploiteur, n'offrant qu'un salaire dérisoire pour de longues heures de labeur. En conséquence, si l'on souhaite aider les femmees à gagner leur vie, la première question à poser est en fait: "Existe-t-il d'autres occupations susceptibles de rapporter de l'argent que les métiers d'artisanat"? Toutes les possibilités devraient être analysées avec attention. Si les travaux manuels semblent être la solution, beaucoup d'autres questions doivent être prises en compte avant de lancer un projet et nous espérons que ce rapport se montrera utile dans cette perspective. 


\section{L'Artisanat: Qu'est ce que c'est?}

Nous pouvons définir les travaux manuels et l'artisanat dont nous parlons comme des activités où les matériaux, outils et techniques disponibles, ajoutés à la créativité et à l'imagination du producteur, servent à fabriquer des objets. Dans certains pays, le terme artisanat regroupe toutes les occupations utilisant des procédés non-mécaniques telles que la préparation de la nourriture, la fabrication de spécialités culinaires ainsi que nombre d'autres activités manuelles. Nous nous en tiendrons ici aux objets à valeur décorative ou utilitaire, une définition communément acceptée dans les pays en voie de développement.

\section{Les Arts Féminins: Rôles Sexuels et Discrimination dans la Production Artisanale}

Les métiers à bonne rémunération tels que le coulage du bronze, la gravure du métal, la joaillerie, la lapidairerie, le soufflage du verre, le tissage de brocards, etc., sont rarement exercés par les femmes. Leurs techniques restent l'apanage des hommes, demeurent étroitement gardées et sont transmises de père en fils. II est rare qu'une fille apprenne ces techniques car il est entendu qu'elle se mariera, quittera sa famille et pourrait, de ce fait, les communiquer à sa nouvelle famille. Habituellement, les femmes accomplissent des travaux en relation avec leur vie domestique, pour répondre à leurs besoins. Parfois, il peut leur arriver de vendre ce qu'elles ont en trop sur les marchés locaux.
Généralement les arts auxquels les femmes sont exposées se voient appelés par oeuphémisme "arts féminins" car ils restent, sous de nombreux aspects, liés aux activités ménagères. Ils comprennent la couture, la broderie, le crochet, le tricot, le tissage, la vannerie, la tapisserie et, dans certaines régions du globe, la poterie. Ceux qui pensent introduire de nouveaux métiers encouragent la teinture, le batik et le macramé.

Si I'on analyse ces travaux, on s'aperçoit que leur "féminité" réside essentiellement dans le fait qu'ils prennent beaucoup de temps, rapportent peu d'argent et ne sont pas facilement élevés à un niveau de qualité justifiant un prix plus important. Rarement s'avèrent-ils être un pas en avant dans une industrie de petite taille, susceptible d'offrir aux femmes des revenus plus conséquents. Quand ces activités sont commercialisées, les hommes s'emparent généralement de la partie la plus rémunératrice du travail. Prenons l'exemple du métier de tailleur: La tâche la mieux payée est la coupe; elle demande une technique spéciale et, dans $90 \%$ des cas, ce sont des hommes qui l'assurent. Le travail plus pénible et moins payé, comme la couture à la main, les finitions et la pose des boutons, est réservé aux femmes et elles touchent les salaires les plus bas.

La situation peut même devenir pire quand ces "arts féminins" sont commercialisés. En Inde, la broderie blanche, "chikanwork", réalisée sur un tissu de coton très fin, représente une des grosses opérations commerciales d'artisanat. Les femmes qui créent ces broderies sont musulmanes et, obéissant à leurs coutumes, restent confinées

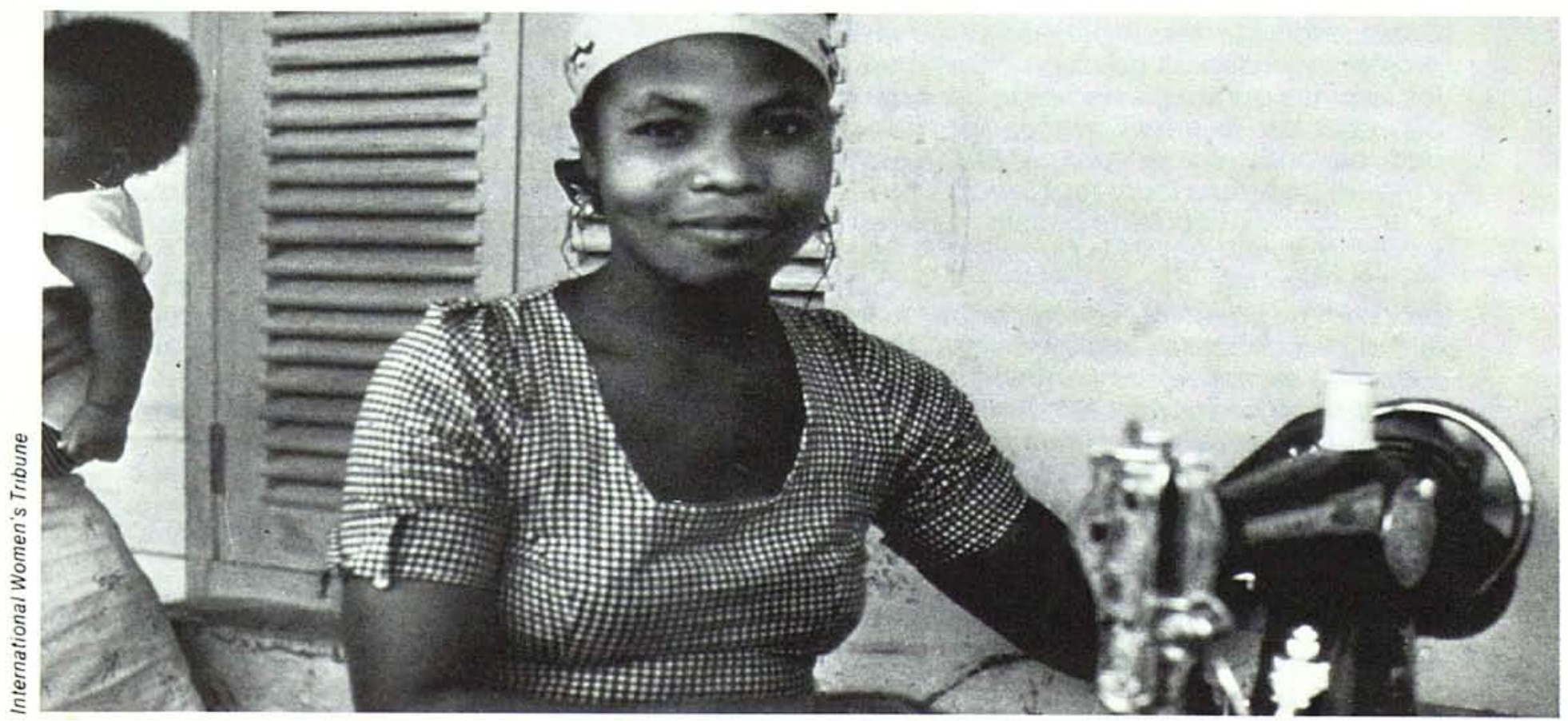




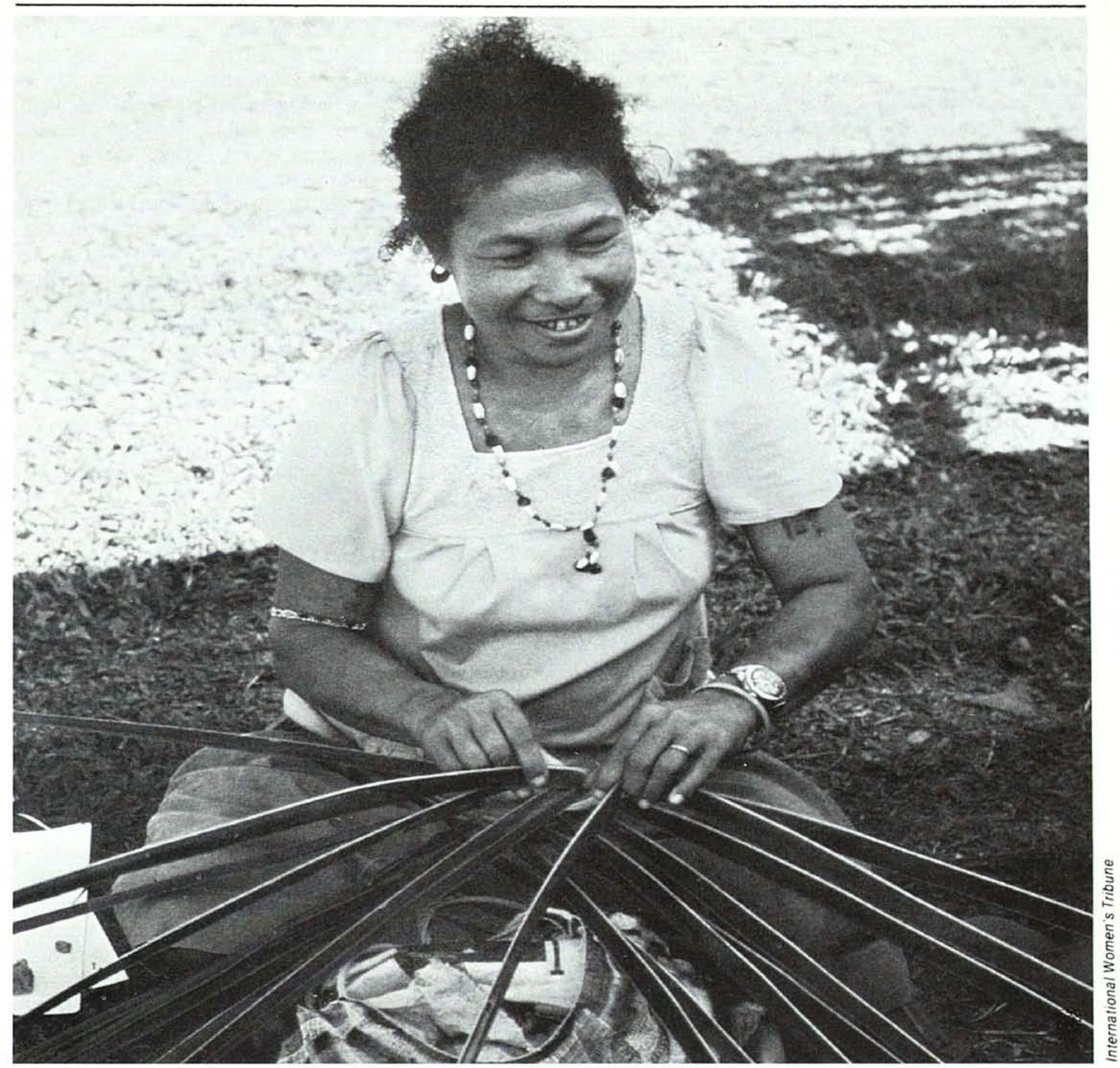

dans leurs maisons. Le travail leur est apporté par des négociants et des intermédiaires. Pour la couture à la main et la broderie d'une chemise, elles touchent un maigre salaire équivalent à 0,25 Dollars US pour huit à dix heures de labeur. Un tel salaire relève de l'exploitation.

\section{Casser Le Mythe des Stéréotypes}

Bien que la plupart des sociétés fassent la distinction entre métiers féminins et métiers masculins, cette division du travail est sans fondement universel. Ce qui est inacceptable dans une société se révèle être pratique courante ailleurs. Par exemple quand nous pensons maréchal-ferrant, nous pensons homme.
Mais n'importe qui ayant voyagé par la route dans le nord de l'Inde a pu voir des femmes travailler le fer, côte a côte, martelant avec un rythme parfait les métaux qu'elles forgent en outils d'agriculture. Dans la plupart des pays d'Asie, ce sont les hommes qui fabriquent la poterie mais pour ceux d'une grande partie de l'Afrique, le simple fait de toucher les instruments de poterie reste tabou. Traditionnellement, les femmes sont supposées faire le tissage mais, dans pratiquement toute l'Afrique, c'est une tâche masculine. Le travail fin, précis et extrêmement lucratif de l'orfèvre appartient au monde masculin mais on pourrait le caractériser comme un art plus doux et les femmes ont prouvé qu'elles pouvaient l'exercer auusi bien. Aujourd'hui, un des 
meilleurs joaillier-orfèvres Turcoman de la région de la mer Caspienne, en Iran, est une femme.

La vérité est que les femmes peuvent exécuter tous les travaux que font les hommes, si tant est qu'elles en aient l'occasion et la formation. Si les travaux manuels et l'artisanat sont destinés à devenir une activite viable et génératrice de revenus pour les femmes, ce genre de distinction doit être dépassé.

\section{La Vie des Femmes de Milieu Rural}

Avant de mettre en place n'importe quelle idée, susceptible d'assurer un revenu aux femmes, il est essentiel d'avoir une connaissance approfondie de la vie de celles que l'on se propose d'aider. Ainsi, ce programme peut vraiment devenir un moyen d' améliorer leur vie et non pas une corvée de plus à supporter dans une existence déjà surchargée.

En milieu rural, c'est généralement la femme qui a la charge des besoins immédiats de la famille. Ses tâches sont nombreuses et elle doit faire face à d'énormes pressions. Elle doit aller chercher l'eau, souvent à de longues distances, amasser le bois et autres combustibles, préparer la nourriture et les repas de la famille. Elles est responsable des petites

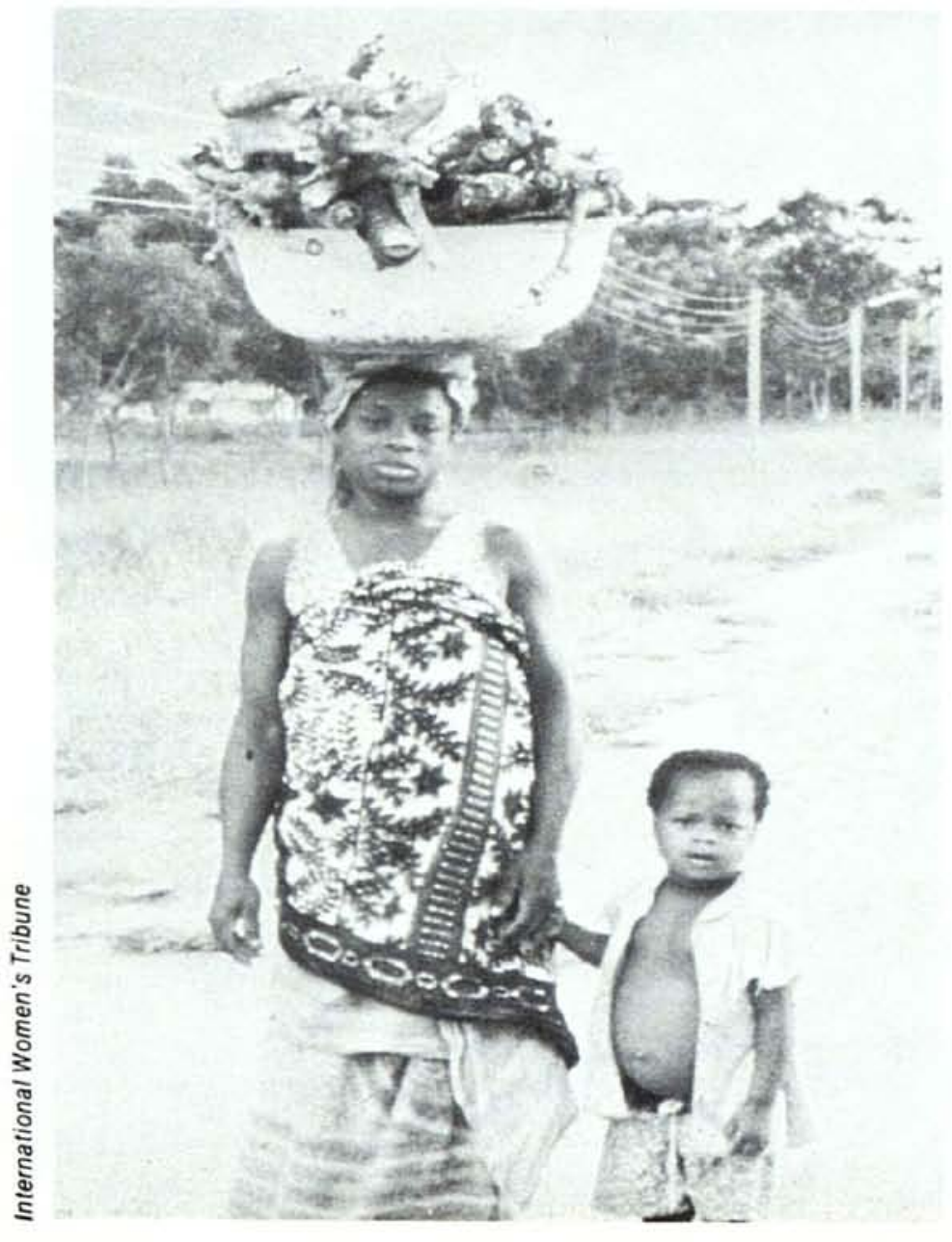

réparations de la maison et essaye de répondre aux besoins du ménage en fabriquant des récipients avec les matériaux disponsibles et en raccomodant les vêtements et les peaux. Le recyclage, un mot tellement à la mode aujourd'hui, est sa spécialité. Les vêtements usés deviennent des morceaux de couverture. Les vieux papiers sont réduits en bouillie et transformés en simples boules de papier-mâché.

Pour lancer de façon effective un programme rapportant à la femme en milieu rural l'argent dont elle a tellement besoin, on doit avant tout examiner sa vie dans son ensemble. Y-a-t-il un moyen d'alléger ses charges et ne devrait-on pas s'occuper de cela d'abord? Parfois, de simples améliorations ou innovations dans les outils et les installations réduisent les corvées et suffisent à lui procurer le temps libre dont elle a besoin pour se livrer à des activités lucratives.

\section{Production Artisanale: Recommendations}

Avant d'essayer de mettre en place un programme de production artisanale dans le but d'assurer aux femmes un revenu, il est absolument primordial de regarder ce qui existe déjà, ce qui leur est accessible, basé sur des techniques traditionnelles ou facilement assimilables. De même, faut-il connaitre et étudier la demande présente pour le produit sur les marchés locaux et dans les régions voisines. En outre, il est nécéssaire de réfléchir à la manière de créer et de diriger les structures d'organisation qui assureront l'encadrement dont le projet aura besoin. Dans certains cas, un programme d'artisanat sera le reponse et dans d'autres, non. Par exemple l'Inde, avec la majorité de sa population en milieu rural et un taux de chômage élevé, a developpé avec succès son artisanat en suivant une politique de protection pendant de nombreuses années et en offrant diverses formes d'assistance technique, y compris une campagne de marketing bien menée, organisée par des compagnies publiques et privées. Cependant, dans d'autres pays ayant une population limitée et différentes possibilités d'emploi, travaux manuels et artisanat ne seront pas une stratégie efficace. Dans certaines régions d'Afrique où la terre est fertile et où, traditionnellement, les femmes cultivent et vendent leurs produits sur les marchés sans être restreintes dans leurs mouvements, l'artisanat n'est sans doute pas la meilleur solution. Mais, dans ce cas, pour accroître leurs revenus, une amélioration de leurs pratiques agricoles et du traitement de la nourriture sera souvent préférable. 


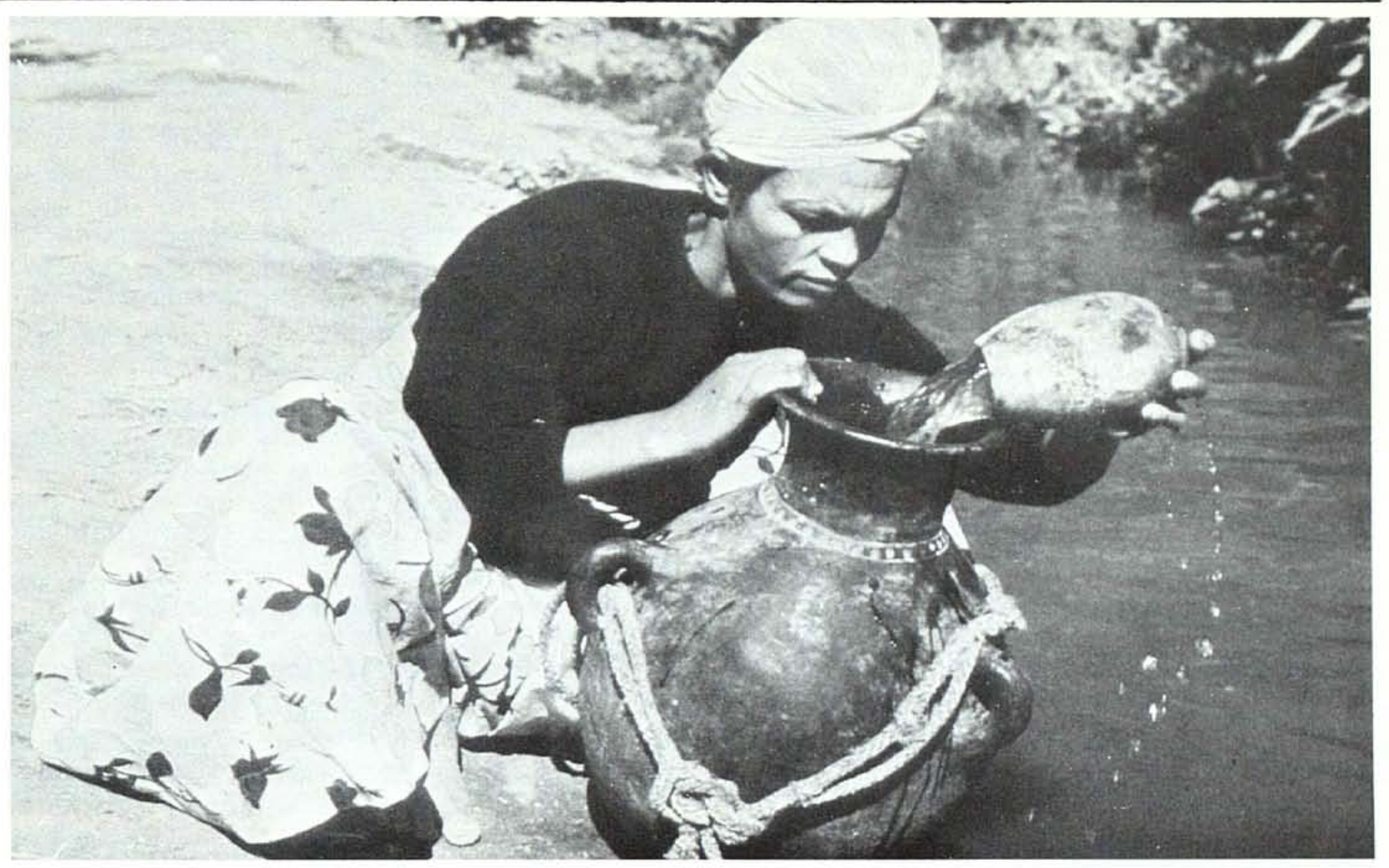

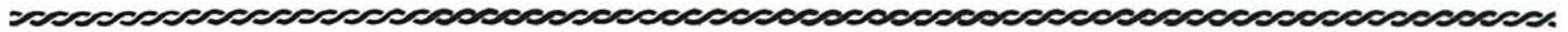

En milieu rural, c'est généralement la femme qui à la charge des besoins immédiats de la famille. Elle doit aller chercher l'eau, souvent à de longues distances, amasser le bois et autres combustibles, préparer la nourriture et les repas de la famille.

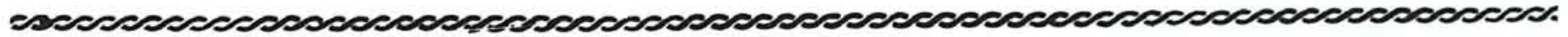

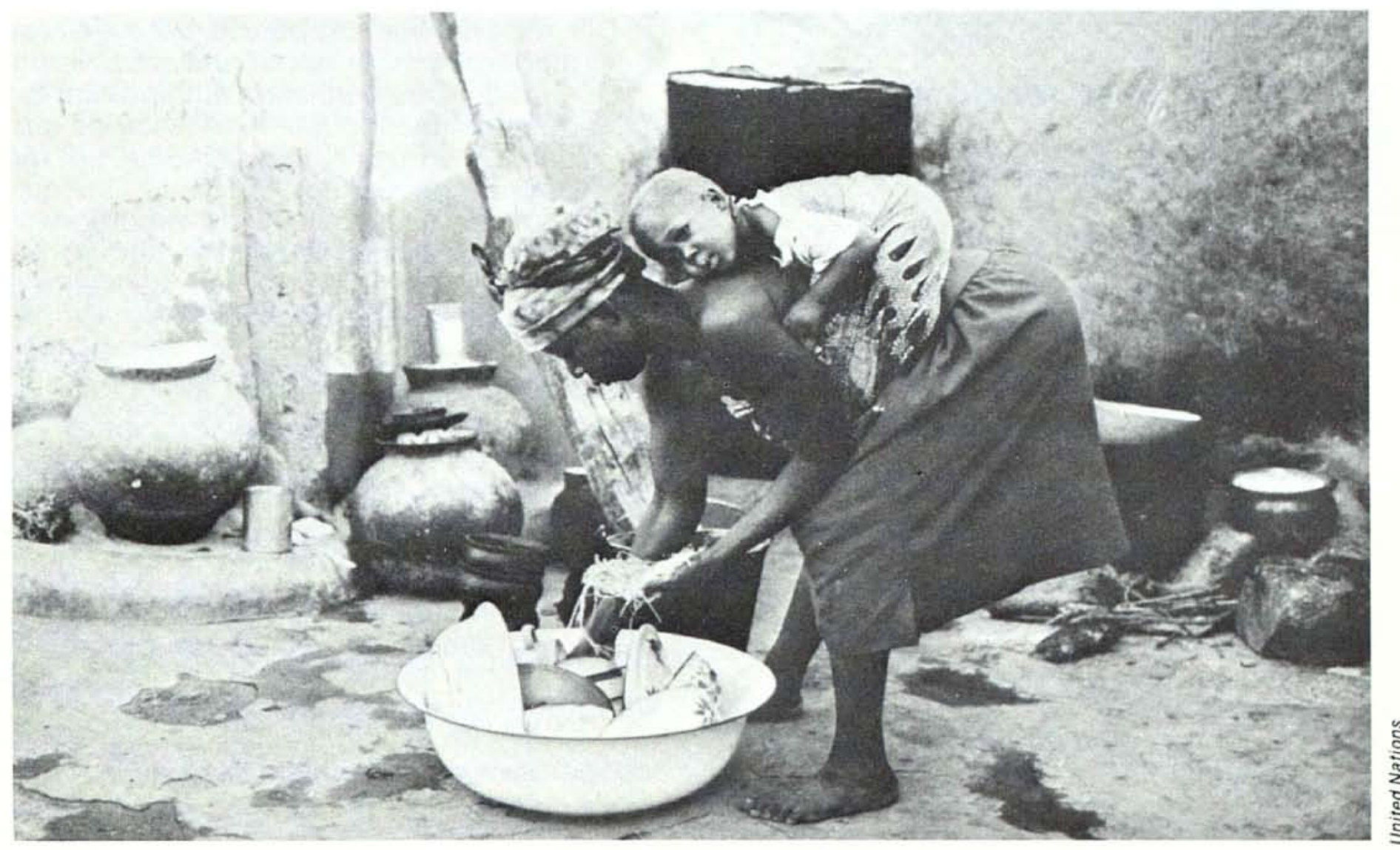


Avant d'organiser un programme d'artisanat, il est recommandé de mener une étude de réalisation.

\section{Etude de Réalisation}

La première étape est d'analyser la situation économique globale, les travaux que les femmes accomplissent déjà, les matériaux qui leur sont disponibles ainsi que les marchés existants et/ou potentiels pour les produits. Dans la plupart des cas, cette étape devrait être confiée à un expert car bon nombre de facteurs sont de nature technique et les jugements requis sont difficiles à atteindre.

Les questions à poser comprennent:

\section{ENVIRONNEMENT ET SITUATION SOCIO-ECONOMIQUE}

1. Quelles sont les femmes disponibles pour un travail de formation et de production (âge, habitation, héritage culturel, etc. . . .)?

2. Font-elles un travail rémunérateur maintenant? Lequel? Travaillent-elles chez elles ou à l'extérieur?

3. Quelles techniques artisanales ou autres talents possèdent-elles qui pourraient engendrer des revenus? (Techniques employées aujourd'hui ou dont elles ne se servent plus.)

4. Ont-elles besoin d'un gagne-pain complet ou simplement de revenus supplémentaires?

\section{INFORMATIONS TECHNIQUES}

5. Quelles sont les techniques utilisées à la production? Décrire les moyens techniques disponibles.

6. Quelles sont les matières premières utilisées dans la fabrication du produit? Sontelles trouvables sur place? Quel en est le coût?

7. Quelles sont les autres matières premières disponibles sur place? Y-sont-elles utilisées? Quel en est le coût?

\section{MARKETING}

8. Où la population locale vend-t-elle ses produits? Comment et quand? A quel prix?

9. Quel est le prix courant pour des types de produits similaires faits à la main ou à la machine?

10. Quels sont les produits en demande dans la communauté qui ne soient pas encore fabriqués par les industries locales?

11. Combien de temps est-il nécessaire pour mettre en place les nouvelles techniques exigées et commencer à produire?

12. Quel marché visons-nous? Local? regional? National? Marché d'exportation?

Voici un cas réel où une étude de réalisation de cette nature a été menée par un spécialiste, conseiller d'une industrie gouvernementale responsable d'un programme de développement rural et non-fermier. Un secteur pauvre de Kirman en Iran fut rapidement étudié et l'on trouva que:

a. C'était une région dont l'activité agricole restait marginale, dépendant de pluies irrégulières. La morte-saison était très longue. Les travaux autres qu'agricoles étaient très limités mais chaque famille possédait des moutons qui donnaient une laine de bonne qualité.

b. Toutes les femmes avaient des métiers à tisser horizontaux et primitifs. Les tapis qu'elles tissaient étaient de piètre qualité mais reproduisaient cependant les motifs traditionnaux des tribus.

c. Les tapis plus vieux et tissés soigneusement sur lesquels figuraient les mêmes motifs se vendaient chers sur les marchés de tapis anciens.

d. On vendait aux marchands des villes la laine d'excellente qualité provenant des moutons et les tisseuses de Kirman ne disposaient plus de bonnes matières premières.

e. II n'existait pas de teinturerie sur place.

f. II n'y avait que très peu de métiers à tisser modernes et ils appartenaient aux marchands. Ces derniers n'employaient que des hommes pour la fabrication de tapis de bonne qualité, très demandés et rapportant beaucoup d'argent. Quelques hommes possédaient leur propre métier et, de ce fait, non seulement gagnaient un salaire mais également bénéficiaient du profit de la vente des tapis.

De plus, l'expert apprit que d'importantes mines de cuivre de la région allaient être exploitées dans les dix années à venir. Beaucoup d'hommes allaient sans doute abandonner le métier à tisser pour les mines et, en conséquence, le niveau des revenus de la région augmenterait, provoquant éventuellement une expansion du marché du tapis. A partir de toutes ces informations, on estima qu'il valait la peine de mettre en place un programme d'amélioration des techniques de tissage et de tapisserie des femmes de Kirman.

L'idée était d'aider celles-ci à former une coopérative qui traiterait la laine de la région. Cette coopérative augmenterait leur production en: 


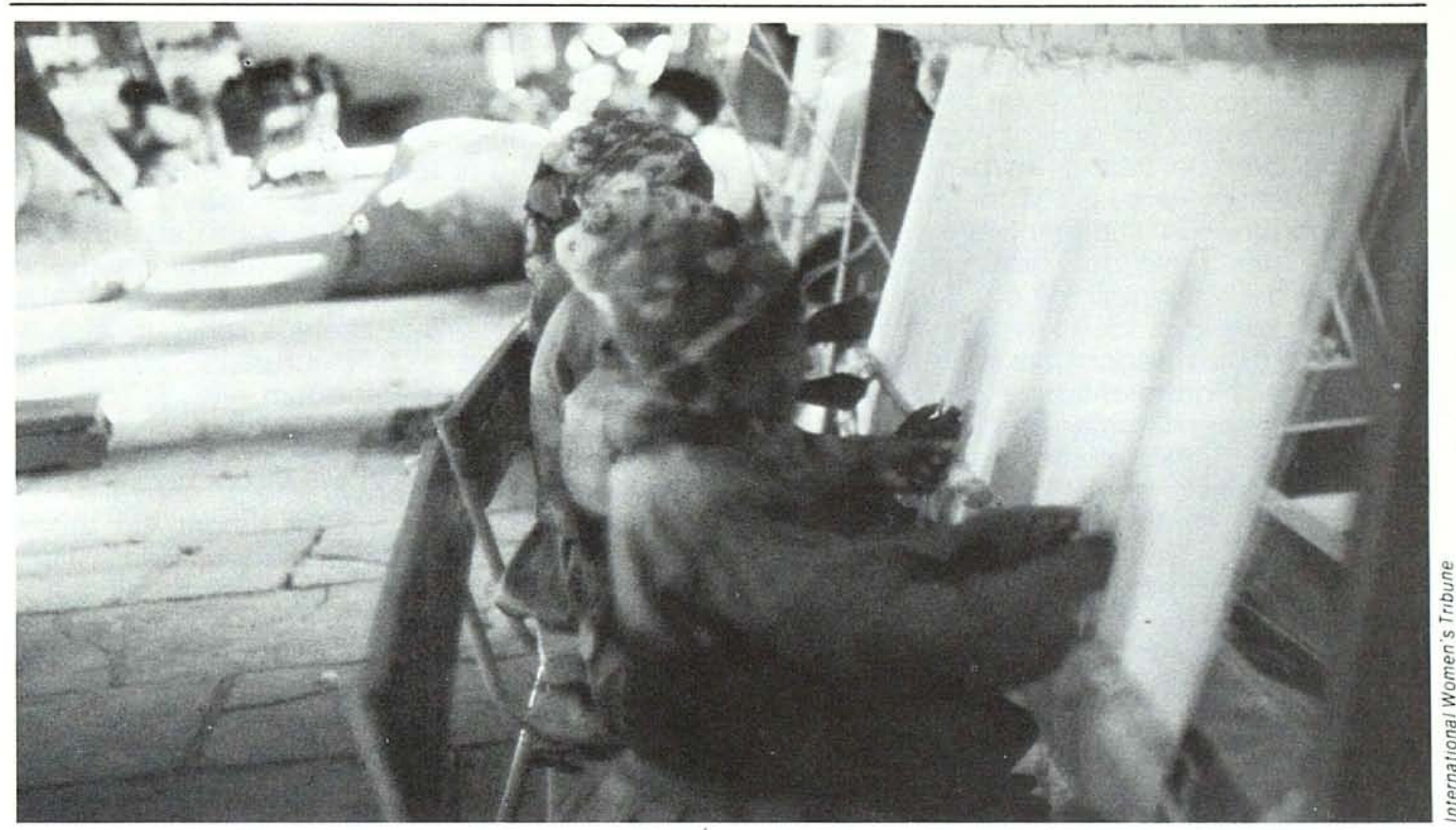

- leur fournissant une matière première de meilleure qualité;

- subventionnant le coût de nouveaux métiers à tisser de façon à ce qu'elles puissent posséder leur propre équipement;

- perfectionnant leur technique pour qu'elles puissent continuer à tisser leurs motifs traditionnaux mais en accroître la qualité.

- enseignant plus tard aux meilleures comment utiliser un modèle pour pouvoir reproduire plus de forme et de couleurs.

Par l'intermédiaire de centres d'économie familiale locaux, les femmes se réunirent et I'on discuta du projet avec elles. On s'aperçut ainsi que les nouveaux métiers à tisser verticaux ne pouvaient être installés à l'intérieur de leurs maisons car les toits étaient trop bas et la lumière insuffisante. On résolut ce problème en suggérant que le montant de la subvention destinée à couvrir le coût du métier à tisser comprenne également le coût du matériel nécessaire pour construire un simple abri, adjacent à la maison, où l'on pourrait installer deux métiers dos à dos. Le gouvernement donnerait poutres, piliers et plaques de métal pour le toit mais les femmes et leur famille devraient construire l'abri elles-mêmes, apportant ainsi leur contribution au projet.

La subvention gouvernementale assura la moitié du coût des métiers à tisser, l'autre moitié étant à la charge des femmes: $10 \%$ en dépôt initial et le solde à payer sur l'année suivante à partir des gains obtenus. Cela était tout à fait raisonnable car on estimait les revenus sur un an à trois fois le coût du métier. Les premiers 10\% (environ 15 Dollars US) représentaient une petite somme qu'elles pouvaient obtenir de leurs familles. Les femmes participèrent au projet avec enthousiasme car le fait de posséder un métier à tisser leur permettait d'occuper une position de force dans l'économie du village et augmentait leur crédit.

Ensuite les coopératives locales leur donnèrent les matières premières, avec des instructions sur les modèles et la qualité. Un maître-tisserand les aidait au montage de la lice et à commencer l'ouvrage puis leur prodiguait des conseils de temps à autre. II habitait le village et les femmes pouvaient aller le voir en cas de difficultés. Un maîtretisserand pouvait s'occuper facilement de quinze métiers à la fois. Quand le tissage d'un tapis était en cours, la coopérative accordait un crédit aux ouvrières pour qu'elles puissent subvenir aux besoins quotidiens de leur maison dont elles avaient la charge: Farine, sel, huile, thé, sucre, lentilles, etc

Lorsqu'un tapis était fini, ce qui prenait de 3 à 7 mois, les femmes pouvaient le vendre soit à la coopérative, soit à un marchand, selon qui offrait le meilleur prix. La coopérative calculait le prix de revient du tapis et payait cette somme immédiatement à la réception de l'ouvrage, après avoir déduit les crédits ac- 
cordés auparavant. Puis le tapis était examiné par un expert qui en déterminait la vraie valeur, la valeur marchande. Souvent ce prix était plus élevé que le prix de revient et, dans ce cas, $50 \%$ du prix de vente, en plus du prix de revient, étaient versés à la femme. Tout cela jouait comme un stimulant et la poussait à produire des tapis de haute qualité. Petit à petit, les femmes finirent par faire partie et compter dans la production générale de tapis. Par la suite, les négociants privés trouvèrent qu'elles se montraient plus dignes de confiance que les hommes, tenaient leurs promesses et travaillaient régulièrement. Ils commencèrent à persuader les femmes des régions voisines à se lancer dans des projets similaires et à entrependre un tissage de qualité. L'émigration des hommes vers les villes, pour trouver de meilleurs emplois, accélera le processus.

Ce programme réussit car il était fondé sur une recherche approfondie des conditions socio-économiques existantes, utilisait les matières premières et les techniques disponibles localement et répondait à la forte demande pour le produit. Le fait de posséder leur propre métier à tisser et de bénéficier à l'origine d'une direction et d'une aide assez souple permit aux femmes d'avoir accès à une activité commerciale aupararant réservée aux hommes. Eventuellement, elles finirent par devenir indépendantes de toute assistance gouvernementale continue.

\section{Diversification des Produits}

II existe d'autres approches permettant d'accroître les revenus tirés de l'artisanat: a) Lancer de nouveaux produits ou modifier un produit traditionnel, sans pour autant changer de façon substantielle les techniques utilisées; b) mettre au point de nouvelles techniques et de nouveaux produits. Dans les deux cas, une étude de réalisation est encore plus importante.

Un exemple, qui illustre le développement de nouveaux produits ou leurs modifications tout en utilisant des techniques existantes, vient d'un village de potiers en Inde où, depuis des années, les familles fabriquaient des jarres à eau pour le marché local. Leurs gains hebdomadaires pour la fabrication de ces articles ne dépassaient jamais 6 Dollars US par famille. L'introduction de nouveaux produits tels que chandeliers, lampes de travail ou de jardin et petits supports perforés, leur apporta non seulement une nouvelle clientèle mais leur permit également de demander un prix plus élevé pour leur travail et d'augmenter ainsi leurs revenus.
Dans un autre cas, des femmes Turcomanes tissaient des pièces de soie superbes mais étroites pour lesquelles n'existait qu'un marché local limité. En changeant le cadre et les peignes des métiers à tisser, elles purent fabriquer des pièces de $90 \mathrm{~cm}$, pour lesquelles existait un marché grandissant, et elles triplèrent leurs gains. Une dépense minimum et à peine une semaine pour pouvoir s'adapter aux nouveaux métiers suffirent à ranimer un artisanat moribond et à assurer une source de revenus pour un important village de tisseuses.

Parfois on peut reconvertir et adapter un art à une ligne entièrement nouvelle de produits. En Ethiopie, récemment, un projet entreprit d'adapter les techniques des potiers traditionnaux qui fabriquaient des jarres à provisions et des récipients pour la cuisine, à la production de matériaux de construction indigènes, briques, tuiles, tuyaux et larges bacs à eau. Cela répondait aux besoins locaux et remplacait les plaques de métal et d'amiante importées. Sans ce changement, les artisans auraient dû rapidement faire face à la compétition du plastique et de l'aluminium renforcé, permettant la fabrication d'ustensiles de cuisine et de conserve plus légers et incassables.

L'autre moyen de diversifier les produits est d'introduire de nouvelles techniques et de nouveaux matériaux. Parmi les exemples où les femmes ont appris un art qu'elles ne pratiquaient pas auparavant, on trouve la taille du verre en Inde, I'impression sur tissu au Kenya, la gravure sur bois et la broderie teinte en Ethiopie.

L'introduction de nouveaux produits exige habituellement de former les femmes à des techniques qu'elles ne connaissent pas, demande un investissement d'importance dans un nouvel équipement, se heurte aux essais et aux erreurs inévitables, recquiert une direction attentive, etc. ... A moins que les produits existants soient nuls ou impossibles à améliorer, cela ne devrait pas être tenté. II est primordial de conduire l'étude de réalisation en accordant une attention particulière à l'étude de marché, la disponibilité des matières premières, l'expertise technique et la direction. Matériaux que l'on peut se procurer sur place sont à utiliser partout où c'est possible et le coût doit rester minimal. Au Bangladesh, l'emploi de simples fibres de jute pour la fabrication de crémaillères, sacs, tapis et autres objets utilitaires, suggeré par une société coopérative venant en aide aux veuves de guerre, remporta un succès sans précédent. Par contre, l'utilisation de matériaux importés ne provoque généralement pas de bons résultats. Au Ghana, le projet de fabrica- 
tion de fleurs, un art traditionnellement riche du pays, illustre un choix malheureux de nouvelles techniques et matériaux. Ce programme échoua car le matériel synthétique, importé à un prix élevé, ne supportait pas l'humidité du climat. Le résultat final était des fleurs artificielles qui se fanaient plus vite que les naturelles!

\section{Marketing}

Le marketing est une étape essentielle dans n'importe quel programme d'artisanat et ses problèmes ont poursuivi la plupart des projets imaginés par les organisations assistant les pays en voie de développement. Souvent, ces associations décident de démarrer un programme sans connaitre la demande des consommateurs, les techniques employées, les prix de vente existants ou les points de vente et de distribution possibles. Il est impératif d'entreprendre une étude de marché détaillée avant de se lancer dans la commercialisation d'un artisanat. Là encore, cette étude devrait être menée par un expert. Certains aspects spécifiques de marketing des produits artisanaux doivent rester présents à l'esprit lorsque l'on veut en faire la commercialisation:

a. On doit fabriquer un produit de haute qualité. Un article mal fini et ne pouvant remplir la tâche pour laquelle il a été crée, trouvera peu d'acheteurs et ne constitue pas une bonne publicité.

b. Le prix d'un article doit être fixé en fonction de la compétition. Un objet que l'on peut trouver moins cher ailleurs ne se vendra pas.

c. II est recommandé d'effectuer un test sur le marché avec des échantillons des nouveaux produits avant de se lancer dans une production à grande échelle.

d. II faut faire tourner ses modèles et toujours avoir de nouvelles créations à offrir. La variété et le changement dans les produits sont essentiels. On doit devancer les besoins et les goûts de ses clients si on ne veut pas se retrouver avec des stocks énormes sur les rayons.

Au Kénya, une association de femmes qui comptent des membres dans tout le pays, Maendeleo ya Wanawake, rassemble plusieurs groupes vivant de l'artisanat. L'association ouvrit une boutique à Nairobi pour vendre leurs produits. Malgré sa situation, dans un quartier commerçant et en face d'un important hôtel pour touristes, la boutique ne remportait pas un grand succès. Les capitaux manquaient pour pouvoir payer comptant au moment de leur réception les produits ex-

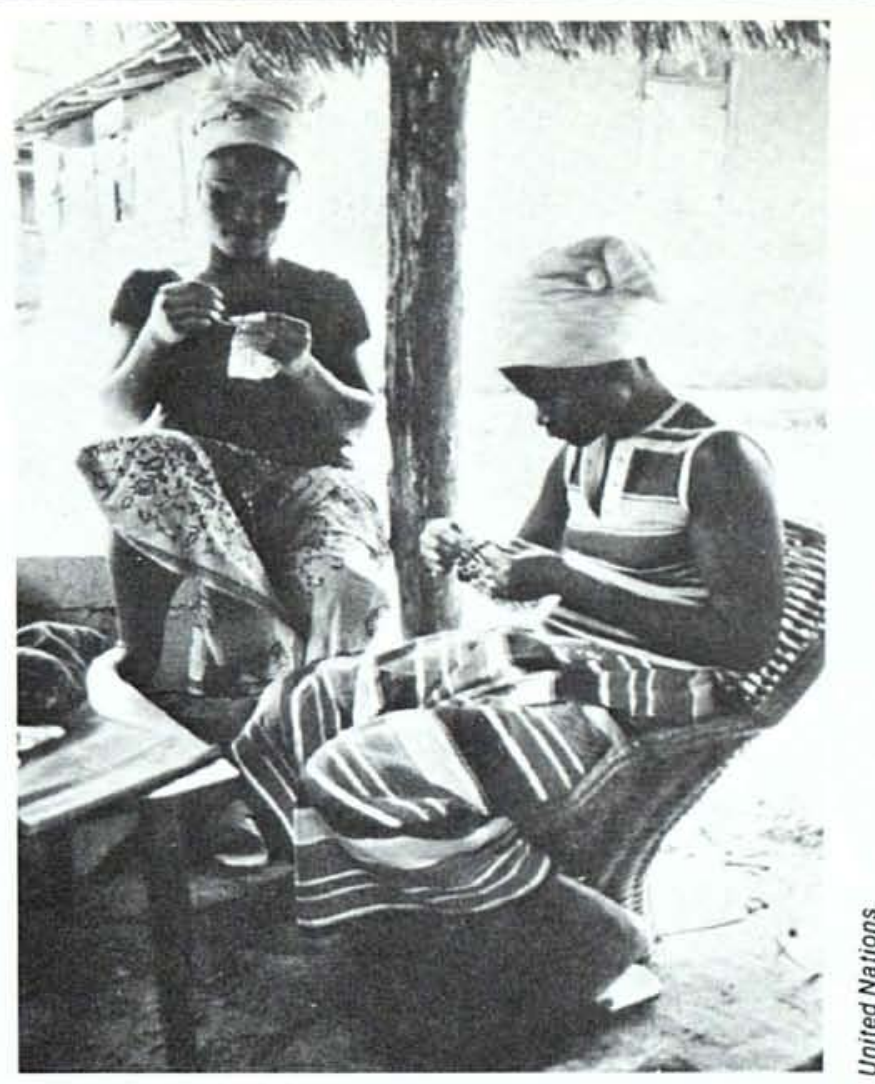

pédiés par les groupes. De plus le manque de capital empêchait l'équipe de marketing d'étudier de nouveaux produits et de créer de nouveaux modèles. Elle était incapable de fournir les matières premières ou d'acheter aux femmes et de payer les produits finis en espèce.

Une agence régionale, assistant les femmes dans le développement d'activités génératrices de revenus, étudia le problème et conclut que le piètre succès initial de Maendeleo ne devait pas être considéré comme un échec. Ils conseillèrent une nouvelle approche qui assurait une réserve de capitaux et permettait en même temps à un créateur de lancer de nouvelles lignes de produits. De plus, cette approche permettait d'avoir un assistant sur le terrain qui aidait les femmes à améliorer leurs techniques pour qu'elles puissent produire une nouvelle catégorie de marchandises. L'élément-clef de cette approche est une équipe technique compétente et très motivée, préparée à un travail de longue haleine et aidant les femmes à s'organiser elles-mêmes en associations, coopératives ou unités de production privées.

Lorsque l'on diversifie et améliore les techniques et que l'on développe de nouveaux marchés, il est très important de ne pas se couper des marchés traditionnaux qui sont les marchés ordinaires. Cela est arrivé souvent et avec des résultats désastreux. Deux cas bien connus se sont produits en Inde. Le premier est l'histoire du "bleeding madras", un coton solide avec des trainées de couleurs délavées, 


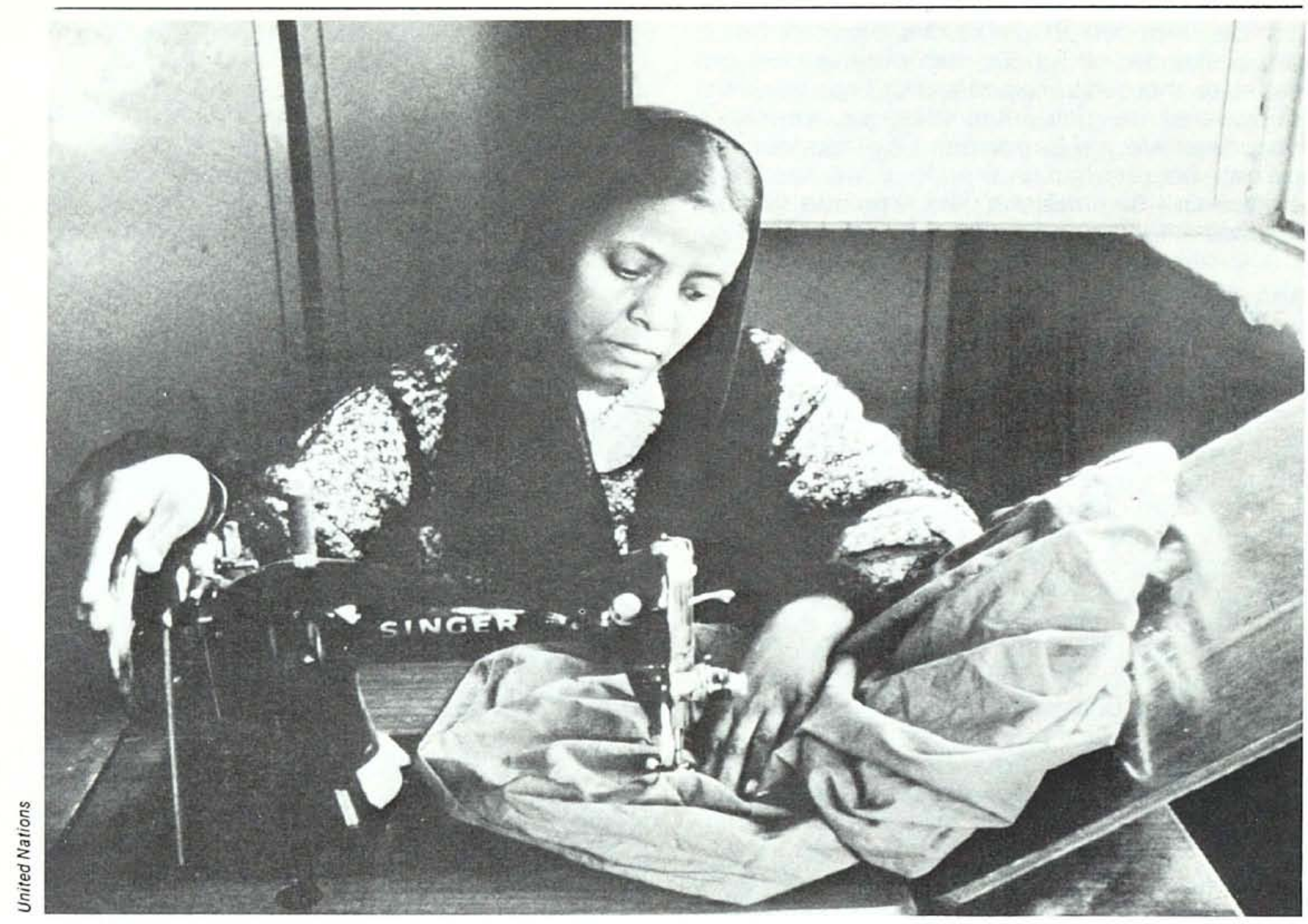

utilisé pour les "lungi" (pagnes d'Hindous), qui devint une mode de courte durée aux Etats Unis. Les artisans des coopératives locales s'orientèrent sur la production de ce nouveau materiel, utilisant des teintures peu tenaces, et ignorèrent le marché traditionnel du sarong, porté par les hommes. Soudain, la demande pour le "bleeding madras" aux Etats Unis s'arrêta et les coopératives se retrouvèrent avec des stocks énormes et pas de marché. Beaucoup tombèrent en faillite et de nombreux artisans connurent la famine.

Dans un autre cas, les fabricants de statuettes de divinités en métal, dans le centre de l'Inde, reçurent pendant deux ans d'importantes commandes du gouvernement pour l'exportation. Pendant ce temps, ils n'approvisionnèrent pas la population des tribus locales qui avaient constitué leur clientèle régulière et avec lesquelles ils maintenaient un lien culturel proche. Ce lien fut rompu et quand le gouvernement cessa ses commandes la troisième année, les travailleurs durent faire face à d'énormes problèmes.

L'expérience montre que l'on devrait concentrer ses recherches pour trouver des marchés à l'interieur du pays, d'abord près des lieux de production et ensuite dans les villes et les régions plus éloignées. Ces marchés locaux sont des points de distributions réguliers et représentent peu de problèmes de transport et de marketing. De plus, le marché ordinaire est un marché qui différencie et rend les préférences des consommateurs faciles à établir. II procure aussi un "feedback" rapide qui aide à mettre en place des standards de production acceptables et maintenir un contrôle de la qualité.

La production d'exportation, d'un autre côté, pose beaucoup de problèmes très difficiles à résoudre. En règle générale, cela exige des niveaux de production importants et réguliers. Les articles doivent être conçus et leurs qualité maintenue de manière à plaire à des gôts étrangers et souvent inconnus. Fréquemment, il faut compter avec des règlements et des lois compliquées, imposés par les gouvernements, sans parler des problèmes de transport. On a donc besoin de resources financières beaucoup plus importantes si l'on veut lancer une opération de marketing pour l'exportation qui soit couronnée de succès.

Lorsque l'on développe des projets pour le marketing de produits artisanaux, les besoins des femmes sont prioritaires mais on 
ne doit pas en oublier pour autant les exigences commerciales. II faut conserver un équilibre entre les activites de développement et les opérations commerciales. Cela recquiert une direction expérimentée. The Central Cottage Industries Emporium, un organisme bien connu de New Delhi, en Inde, représente un excellent exemple de projet de cette nature fonctionnant très bien. Cette organisation fut fondée par un groupe de travailleurs sociaux, très motivés, bénéficiant de l'assistance du gouvernement. On appliqua de facon systématique de solides pratiques commerciales. On aida de nouveaux et plus anciens centres de production dans la création de nouveaux produits. Des procédures pour le contrôle de qualité furent mises en place et des campagnes de promotion des ventes furent lancées. Leurs efforts aboutirent non seulement à la création d'emplois pour des femmes aux revenus faibles mais aussi à la meilleure qualité d'articles. Leur boutique de produits artisanaux est actuellement la meilleure en Inde.

\section{Support Institutionnel: Ressources et Protection Financières}

Dans les organisations féminines, indépendantes ou gouvernementales, il y a une tendance à mettre en place des activités de façons diverses et sans les rattacher aux institutions existantes qui pourraient aider au développement de programmes d'apprentissage, en marketing et en argent. L'utilisation de telles structures peut mener à des programmes très réussis. Par exemple le gouvernement Tunisien a étudié toute une série de politiques d'assistance et de protection des petites entreprises dans le cadre de son nouveau plan de décentralisation, qui accorde des avantages spéciaux aux entrepreneurs dûment qualifiés. Le programme Tunisien "Familles dans la Production" a démontré une utilisation créative de ces politiques. Ce projet fut organisé par le Ministère des Affaires Sociales en collaboration proche avec le Centre de Promotion de l'Artisanat, un autre organisme gouvernemental. Ensemble, ils sélectionnèrent un art à développer et un stage d'apprentissage fut financé et mis en place par le Ministère des Affaires Sociales. L'instructeur, les matières premières et les modèles étaient fournis par le Centre de l'Artisanat, assurant de cette façon le niveau de formation nécessaire pour poursuivre une production commerciale.

Pendant les neuf mois d'instruction, les stagiaires recevaient de l'argent de poche de manière à être capable financièrement de participer au programme de formation. De plus le stage était conçu pour permettre aux participantes de travailler indépendemment dès la fin du programme. Les neuf mois étaient divisés en deux périodes: Pendant les cinq premiers mois, les stagiaires travaillaient sous le même toit et sous supervision stricte. Pendant les quatre mois restant, on installait outils et équipement dans leurs maisons et

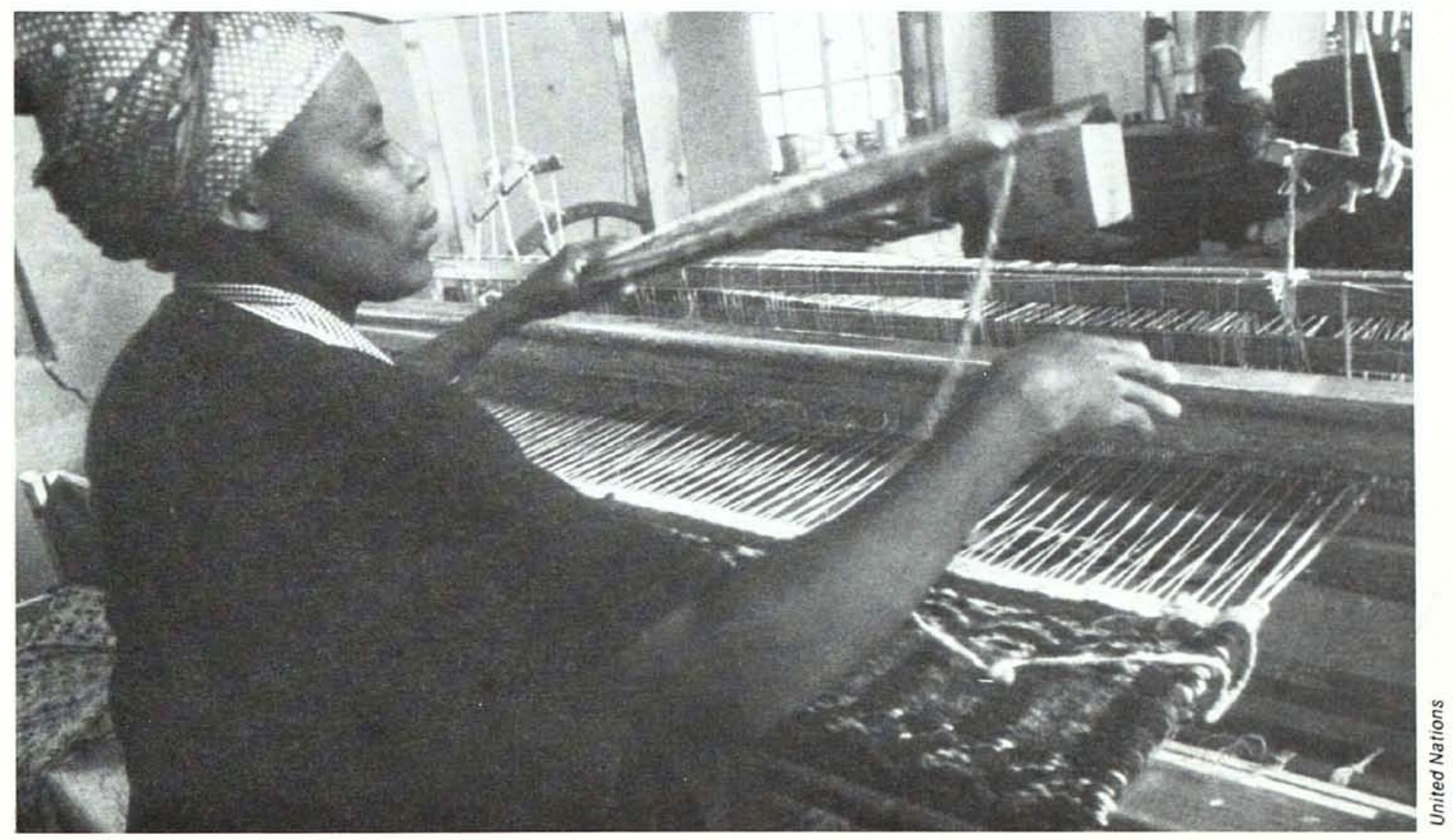


elles apprenaient à travailler seules. Une fois indépendantes, elles restaient en contact avec le département commercial du Centre et pouvaient vendre leurs produits soit par son intermédiaire, soit à des marchands. La collaboration avec un organisme spécialisé en place empêcha le programme d'essuyer les échecs habituels, provoqués par un pauvre niveau technique et une piètre production et qui, à leur tour, entrâinent des problèmes de marketing et d'exploitation par les marchands. Ces problèmes sont malheureusement trop communs pour la plupart des centres d'artisanat créés pour les femmes.

\section{Stratégie D'Organisation - Dynamique de Groupe}

Un autre élément important lorsqu'on prépare un programme auquel les femmes participent et dont elles bénéficient, est de posséder une solide structure d'organisation. Qu'il s'agisse de coopératives, d'associations, de sociétés reconnues ou de groupes traditionnaux, il est essentiel d'aider les femmes à s'organiser en unités économiques viables pour éviter l'exploitation. Lorsqu'elles ont affaire à des groupes organisés, les associations gouvernementales et indépendantes peuvent apporter une aide qu'elles ne peuvent offrir à des familles éparses ou des particuliers. De plus, les organisations officiellement reconnues peuvent recevoir des fonds et accorder des prêts en fonction des besoins respectifs de leurs membres, là où les conditions des prêts individuels sont souvent beaucoup plus strictes et exigent des garanties.

Un des moyens les plus efficace de bâtir une organisation est de la fonder sur des associations féminines traditionnelles. Ces dernières sont de formes variées. L'une des plus courantes reste l'association économique, comme le tontin des vendeuses de marché au Togo ou l'arisan en Indonésie. Les femmes s'y rencontrent périodiquement et, à chaque réunion, versent une somme d'argent. Le montant recueilli est remis à chaque membre à tour de rôle. Chaque femme peut ainsi disposer d'un capital qu'elle ne trouverait pas en temps normal et qui lui permet d'effectuer des investissements importants comme des réparations sur la maison, l'achat de grosses quantités de biens en solde, l'acquisistion d'outils, etc ... Quand ces groupes fonctionnent bien, ils peuvent même servir de garantie si quelqu'un souhaite faire un emprunt auprès d'une agence extérieure. Ils peuvent faire pression pour obtenir le remboursement d'un prêt et, si un des membres, pour une raison imprévisible, ne peut assurer un payement, les autres mettront leurs ressources en commun pour rembourser un prêt dans le délais recquis.

Malheureusement, la plupart des projets de développement ignorent les groupes sociaux traditionnaux et tentent d'implanter de nouvelles structures. Souvent, l'acceptation de nouveautés et un succès durable seraient obtenus plus facilement si ces projets essayaient de renforcer les associations en place et leur faire prendre en charge les différentes activités. La coopérative suivant le modèle occidentale reste le type de structure institutionnelle le plus souvent adopté en rapport avec les programmes de développement de l'artisanat. Cela implique que l'on introduit un système qui exige des traces détaillées de toutes les activités entreprises, demande à ses dirigeants un haut niveau d'éducation. Comme dans beaucoup de parties du monde les femmes sont soit analphabètes, soit éduquées de facon marginale, la direction de ces groupes retombe très souvent aux mains des hommes. II n'est donc pas difficile à comprendre pourquoi les femmes qui font partie de telles organisations s'en désintéressent assez vite. Si on veut réussir à améliorer la vie des femmes avec des coopératives, on doit soit en simplifier les procédures ou soit fournir une aide spécialisée à leurs directions.

Par exemple, en Iran, on utilisa les services d'un expert pour participer à la mise en place et veiller à la bonne marche de coopératives de femmes en milieu rural. Les intéressées se faisaient aider pour la comptabilité, les transactions financières complexes, l'achat des matières premières et la vente des produits finis. L'organisateur servait également de lien entre la société et le groupe de marketing de la coopérative. C'est un modèle qui a très bien marché en Iran et qui pourrait être appliqué à d'autres groupes de femmes connaissant les mêmes besoins.

Une autre méthode, développée en Inde, consiste à utiliser une aide abondante à la direction lors de la formation de la coopérative pour la réduire ensuite petit à petit dans les premières années. A la fin de cette période, la coopérative comptera suffisement de membres capables d'assurer son fonctionnement ou sera en mesure de s'attacher les services d'un professionnel. La méthode la plus efficace serait sans doute une combinaison des deux précédentes, en y ajoutant un programme de formation aux tâches de direction. Certains membres recevraient une formation courte et intense qui les préparerait à tenir un poste de responsabilité au sein de l'organisation. 


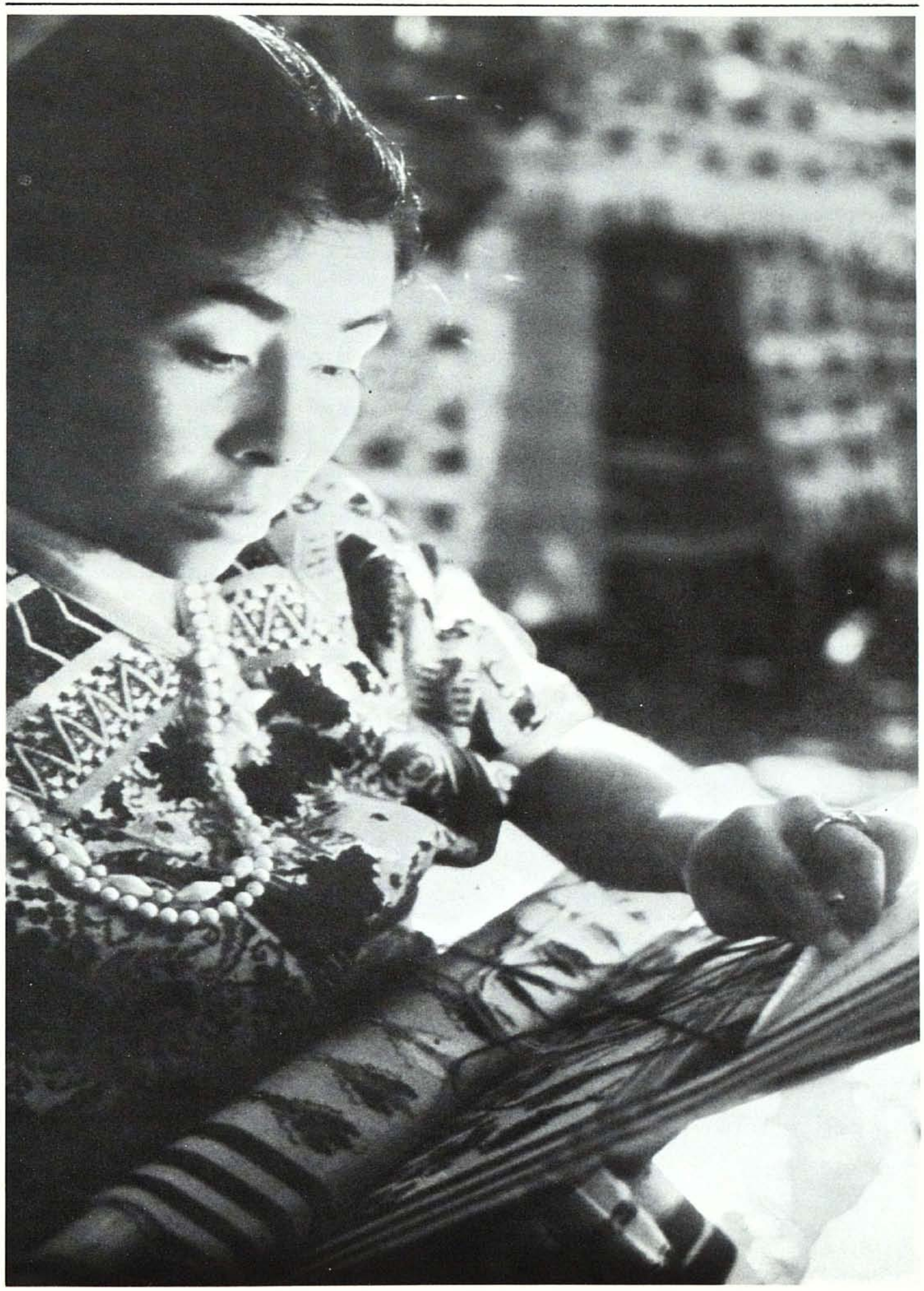


Nous devons souligner ici que les coopératives ne sont pas la seule et unique solution, Au début, les femmes préfèreront peut-être choisir un type d'organisation moins structurée. II existe de nombreux intermédiaires dont on peut se servir avantageusement avec, entre autre, la location en commun d'un espace, l'achat communautaire de matériel ou une structure d'organisation qui garantisse un salaire minimum et une répartition des profits possible à la femme. La forme adoptée devrait être celle qui répond le mieux aux besoins et aux ressources des intéressées.

De plus, on doit se souvenir que ces associations de femmes ne sont pas seulement un moyen de faire marcher des entreprises commerciales. Elles remplissent aussi une fonction sociale où chaque participante sent qu'elle fait partie d'une communauté. Dans des régions où la coutume ne permet pas aux femmes de se livrer à des activités en dehors de la maison ou parmi des hommes, une association féminine peut représenter un premier pas vers le monde extérieur. Ses membres peuvent y rencontrer d'autres femmes aux expériences différentes et participer à des activités qui leur permettent de sortir du monde en vase clos dans lequel elle vivent.

\section{Conclusion}

Les travaux manuels et l'artisanat peuvent être un moyen pour les femmes d'accroître leurs revenus dans certains environnement, mais seulement sous les conditions que nous venons de voir, car ces arts demeurent des spécialités qui ne possèdent que des marchés limités et n'offrent qu'un potentiel restreint d'emplois. Les remarques suivantes résument quelques uns des points-clefs dont on doit tenir compte si l'on veut mettre en place un programme d'artisanat pour les femmes:

1. Les arts "féminins" prennent essentiellement beaucoup de temps, rapportent peu d'argent et offre peu de possibilité de progression technique.

2. II faut donc, avant de mettre en place un programme, étudier les conditions présentes dans lesquelles les femmes travaillent ainsi que les possibilités qui leur sont accessibles en fonction de techniques traditionnelles ou facilement assimilables. II faut également analyser les différentes manières de créer et de diriger les structures d'organisation nécessaires au support d'une activité artisanale. On doit se souvenir que l'artisanat reste souvent un moyen de rapporter de l'argent aux femmes plus

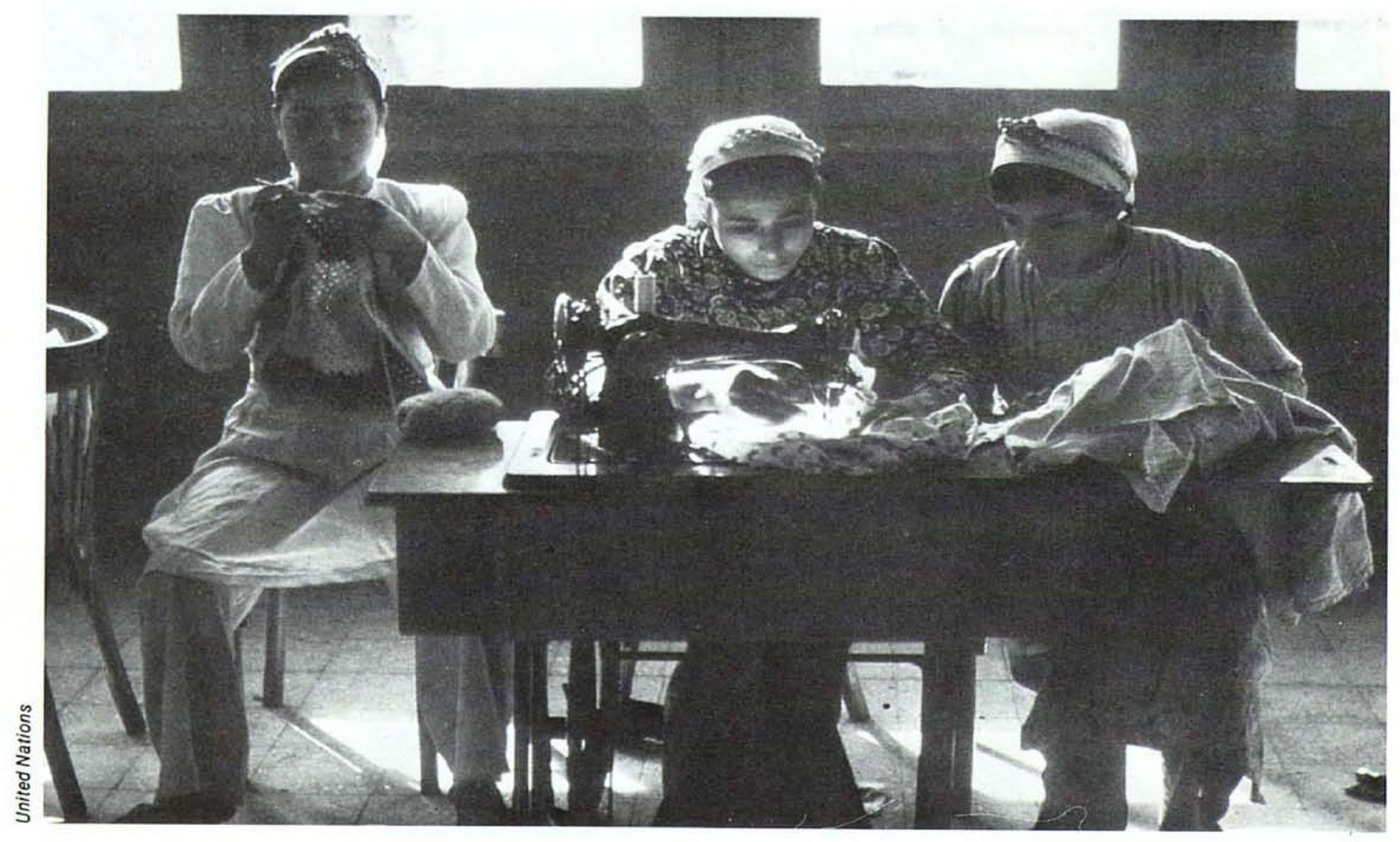




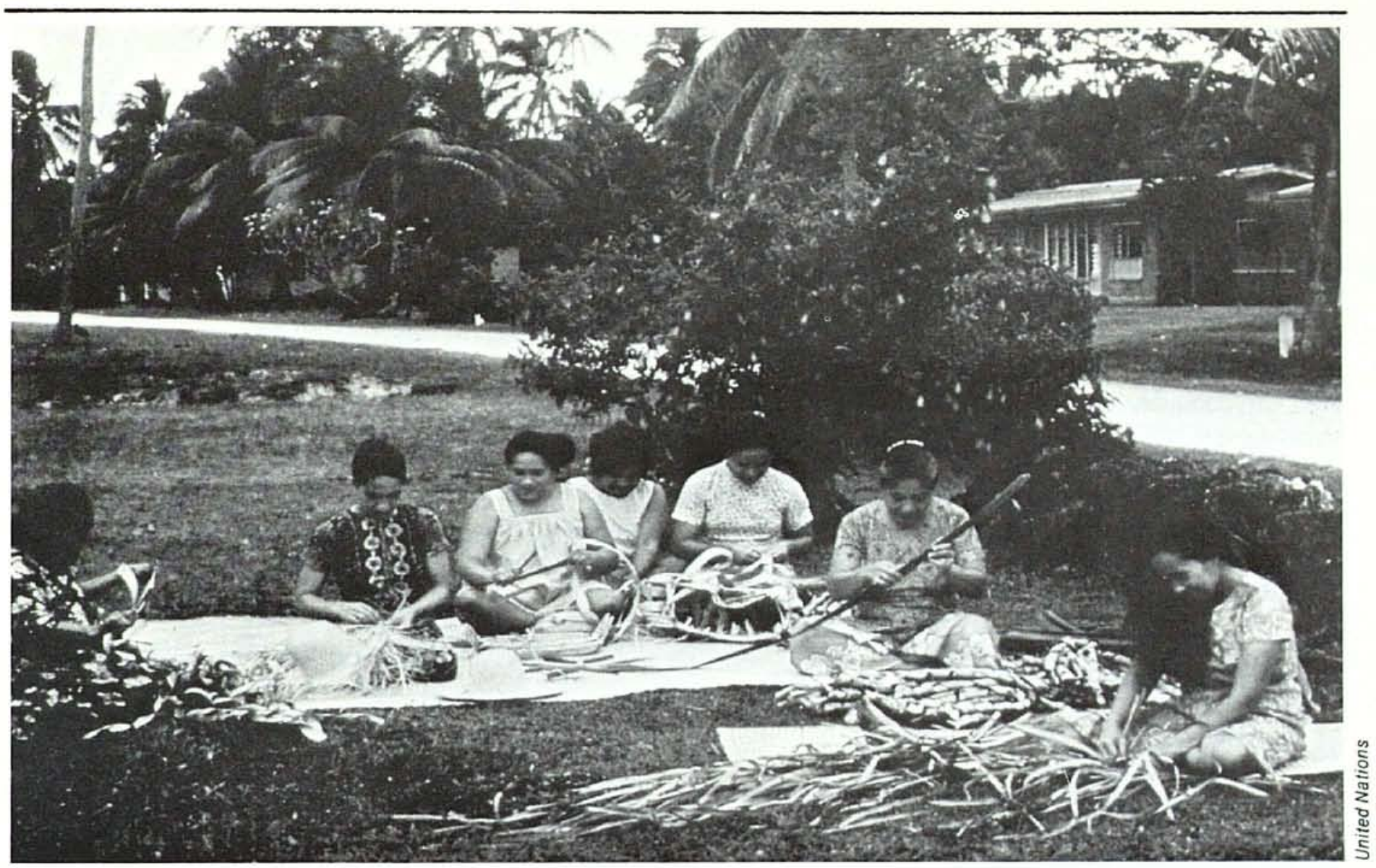

compliqué que d'autres choix comme le traitement de la nourriture sur les marchés locaux.

3. Lorsqu'on pense à des activités génératrices de revenus pour les femmes, il ne faut pas avoir peur de faire preuve de créativité et d'imagination. Les femmes peuvent exercer tous les métiers que font les hommes, si on leur en assure la formation et le marché de l'emploi!

4. Avant toute activité commerciale, une étude de marché est une nécessité absolue.

5. Il faut prendre garde à ne pas rompre les liens avec les marchés traditionnaux, qui sont les marchés ordinaires, quand on recherche de nouveaux points de vente.

6. Les éléments clefs d'un projet réussi sont une équipe motivée et compétente, une direction efficace, capable de prodiguer les conseils nécessaires, d'aider à la diversification, de maintenir une ligne de produit de qualité et de surveiller le marché.
Lorsqu'on touche au domaine de l'artisanat, la prudence est de rigueur. II peut être un 'moyen d'assurer des revenus décents mais peut aussi tourner à l'exploit ation, alourdissant davantage les charges que les femmes doivent déjà supporter et les privant de l'opportunité de rehausser leur position économique et sociale. 


\section{Appendice}

Sources d'information sur des programmes

d'artisanat régionaux et aides techniques.

\section{Organisations régionales offrant une aide technique:}

\section{Afrique:}

African Training and Research Center for

Women (ATRCW)

United Nations Economic Commission for

Africa

B.P. 3001

Addis Ababa, Ethiopie

A l'attention de: Mary Tadesse

\section{Asie:}

Women's Programme Centre

ESCAP

U.N. Building

Rajdamnarn Avenue

Bangkok, Thailande

A l'attention de Daw Awe

All India Handicrafts Board

Government of India

Ramakrishna Puram

New Delhi, Inde

\section{Amérique Latine}

Women's Programme Unit

CEPAL

Castilla 179/D

Santiago, Chili

A l'attention de: Erma Garcia-Schfardet

\section{Caraibes}

Women in Developpement Unit

Extra Mural Centre

University of the West Indies

Pinelands

St. Michael, Les Barbades

A l'attention de: Peggy Antrobus

Women in Development

6 Bartletts

Christchurch, Les Barbades

A l'attention de: Lynn Allison

\section{International}

Organisation Internationale du Travail

$\mathrm{CH} 1211$

Geneve 22, Suisse

Centre de Commerce International

$\mathrm{CH} 1211$

Geneve 22, Suisse

World Crafts Council.

20 West 55th Street

New York, New York 10019 USA

\section{Aide technique-Conseils}

Consultants in Development

2130 P. Street, N.W.

Suite 803

Washington, D.C. 20037

A l'attention de Maryanne Dulansey

(Etudes de réalisation, conseils, étude de marché et développement de produits; services rendus contre rémunération.)

\section{Documentation et Littérature}

"Third World Producers' Guide to Alternative Marketing." de David Dichter

Pour information, écrire à:

David Dichter \& Associates

9 Rue de Vermont

1202 Geneve, Suisse

"Notes on the International Workshop on Alternative Marketing Organizations and Third World Producers" (Septembre 3-8, 1976, Pays Bas)

Pour information, écrire à:

Stichting Ontwikkelings Samenwerking

Kerkrade-Nederland

Holzstraat 19

Revues éditées par Consultants In

Development (CID)

"Expanding the External Market for Third

World Crafts: The Role of Alternative

Marketing Organizations" (existe en Anglais,

Espagnol et Français).

"Formats to Evaluate The Feasibility of Developing Small Industry Projects" (existe en Anglais, Espagnol et Français).

"Craft Item Information Form" (existe en

Anglais, Espagnol et Français).

"Manuel, séminaire/atelier sur l'artisanat"

Toutes les publications du CID sont à vendre.

Dans certains cas, CID se réserve la possibilité d'échanger ces publications contre des documents Equivalents, issus des pays du tiers-monde.

Pour information, écrire à:

Maryanne Dulansey

Consultants In Development

2130 P. Street, N.W.

Washington, D.C. 20037 USA. 


\begin{abstract}
Mise en page: John Cotterman
Typographie: Village Type and Graphics

Photo de Couverture: United Nations

Imprimeur: Graphic Impressions, Inc.

Traduction: Philippe Bette
\end{abstract}

\begin{abstract}
Jasleen Dhamija est directrice du Département des Petites Industries et de l'Artisanat chez les Femmes Africaines, un organisme mis en place par l'Organisation Internationale du Travail et la Commission Economique Africaine des Nations Unis. Sa carrière débuta en Inde il y a 26 ans, où elle joua un rôle de pionnier dans le developpement de l'artisanat et des industries rurales. Elle a également travaillé en Iran pendant 6 ans sur des activités rurales et non-agricoles et à L'université Farabi où elle participa au développement, d'un programme sur les arts appliqués et la culture d'Asie. Elle s'intéresse profondément au développement rural et à l'art populaire. Elle a écrit de nombreux livres sur ces sujets.
\end{abstract}

Nous attendons vos remarques, commentaires et idées de projets à publier dans les prochains numéros de SEEDS. Si vous souhaitez recevoir des exemplaires supplémentaires de ce numéro ou faire partie de nos abonnés, n'hésitez pas à nous écrire. Envoyez votre courrier à:

Ann Leonard, éditeur

SEEDS

P.O. Box 3923

Grand Central Station

New York, New York 10163 U.S.A. 


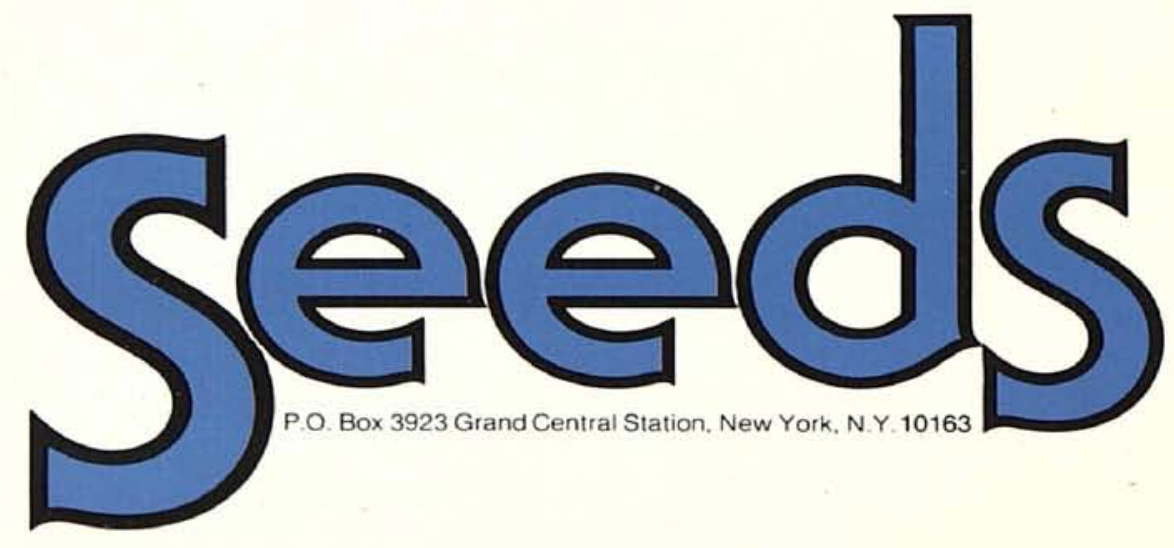

